\title{
Risk Adjusted Output Feedback Receding Horizon Control of Constrained Linear Parameter Varying Systems
}

\author{
M. Sznaier ${ }^{1}$ \\ C. M. Lagoa $^{2}$ \\ N. Ozay ${ }^{1}$
}

\begin{abstract}
In the past few years, control of Linear Parameter Varying Systems (LPV) has been the object of considerable attention, as a way of formalizing the intuitively appealing idea of gain scheduling control for nonlinear systems. However, currently available LPV techniques are both computationally demanding and (potentially) very conservative. In this paper we propose to address these difficulties by combining Receding Horizon and risk-adjusted techniques. The resulting controllers are guaranteed to stabilize the plant and have computational complexity that increases polynomially, rather than exponentially, with the prediction horizon.
\end{abstract}

\section{INTRODUCTION}

A widely used practice to handle nonlinear dynamics is to linearize the plant around several operating points and then use gain-scheduled linear controllers. However, while intuitively appealing, this idea has several pitfalls [17]. Motivated by these shortcomings, during the past few years considerably attention has been devoted to the problem of synthesizing controllers for Linear Parameter Varying Systems, where the state-space matrices of the plant depend on time-varying parameters whose values are not known a priori, but can be measured by the controller. This research has resulted in controller synthesis methods guaranteeing worst case performance bounds (for instance in an $\mathcal{H}_{2}$ or $\mathcal{H}_{\infty}$ sense, see e.g. [1] and references therein). While successful in many situations, these techniques are potentially very conservative in others, since they are based on sufficient conditions,. In addition, these methods are computationally very demanding, requiring the solution of a set of functional matrix inequalities. Obtaining computationally tractable problems requires using both finite expansion approximations as well as a gridding of the parameter space, leading to further conservatism.

The present paper seeks to reduce both the computational complexity and conservatism entailed in currently available LPV synthesis methods by combining risk-adjusted (see eg. [4], [5], [14]) and Receding Horizon (see [13] and references therein) ideas. Our main result shows that, by searching over a set of closed-loop strategies the problem can be reduced to finding a solution to a finite set of Linear Matrix Inequalities (LMIs). Finding the exact solution to this problem has computational complexity that grows exponentially with the horizon length. To circumvent this difficulty, we propose to use a stochastic approximation algorithm that is guaranteed to converge to the solution with probability one, and whose

1 ECE Department, Northeastern University, Boston, MA 02115, msznaier@ece.neu.edu, ozay.n@neu.edu

2 EE Dept., The Pennsylvania State University. University Park, PA 16802, USA, lagoaeengr.psu.edu computational complexity grows only polynomially with the horizon.

The paper draws inspiration, in addition to [19], [21] and [9], from [15], [7] and [3]. The main difference with [15] and [7] is the use of Receding Horizon techniques. Compared with [3], we consider the case of case of LPV dynamics and we obtain a controller that minimizes the worst case performance over all trajectories compatible with the current parameter value. Finally, the use of closedloop strategies, based on the solution of a set of LMIs, results in substantial reduction of computational complexity. In this sense the work presented here is related to the earlier work in [22] advocating the use of Riccati based receding horizon controllers for $\mathcal{H}_{\infty}$ control of Linear Time Varying systems, and to the work in [12], proposing an LMI-based optimization of closed-loop control strategies.

\section{Preliminaries}

\section{A. The LPV Receding Horizon $\mathcal{H}_{2}$ Control Problem}

In this paper we consider discrete time LPV systems of the form:

$$
G\left\{\begin{aligned}
x(t+1) & =A[\rho(t)] x(t)+B_{1}[\rho(t)] w(t)+B_{2}[\rho(t)] u(t) \\
z(t) & =C_{1}[\rho(t)] x(t)+D_{12}[\rho(t)] u(t) \\
y(t) & =C_{2}[\rho(t)] x(t)+D_{21}[\rho(t)] w(t)
\end{aligned}\right.
$$

where $x \in R^{n_{x}}, u \in S_{u} \subseteq R^{n_{u}}, z \in R^{n_{z}}, y \in R^{n_{y}}$ and $w \in R^{n_{w}}$ represent the state, control, regulated variables, measurements available to the controller and a white Gaussian exogenous disturbance, respectively, $S_{u}$ is a convex set containing the origin in its interior, $\rho$ denotes a vector of time-varying parameters that can be measured in real time, and where all matrices involved are continuous functions of $\rho$. Further, we will assume that the set of parameter trajectories is of the form:

$$
\mathcal{F}_{\Theta}=\{\rho \in \mathcal{P}: \rho(t+1) \in \Theta[\rho(t)], t=0,1, \ldots\}
$$

where $\mathcal{P} \subset R^{n_{\rho}}$ is a compact set and $\Theta: \mathcal{P} \rightarrow \mathcal{P}$ is a given set valued map. Our goal is to, given the present value $\rho(k)$ of the parameter vector, find an output feedback control law:

$$
u(k)=f[y(k), y(k-1), \ldots, \rho(k), \rho(k-1), \ldots] \in S_{u}
$$

that minimizes, in some sense, the effect of the disturbance $w$ on the performance output $z$. In the case of LTI systems, this leads to the well known $\mathcal{H}_{2}$ control problem. In the case of Linear Time Varying systems, one could proceed as in [6] 
by considering the performance index

$$
J \doteq \lim _{T \rightarrow \infty} \mathbf{E}\left\{\frac{1}{T} \sum_{t=0}^{T-1} z^{T}(t) z(t)\right\}
$$

where $\mathbf{E}\{\cdot\}$ denotes expected value. Note however, that such a performance index takes into account only the steady state behavior of the closed-loop system. Moreover, since the present value of the time-varying parameter typically does not provide information on the value of the parameter as $t \rightarrow \infty$ (assuming that all of the parameter set $\mathcal{P}$ is reachable from any starting value), robustly optimizing an index of the form (3) entails considering a potentially very conservative double worst-case scenario: worst case trajectory and worse case initial condition in $\mathcal{P}$. Improving both the transient and steady-state behavior while minimizing conservatism suggests the use of a performance index of the form:

$$
J \doteq \mathbf{E}\left\{\frac{1}{N} \sum_{t=0}^{N-1} z^{T}(t) z(t)\right\}
$$

where the horizon $N$ is a design parameter. In turn, this leads naturally to the following Receding Horizon formulation:

Problem 1: Given an LPV system of the form (1), find an output feedback control law:

$$
u(k)=f[y(k), y(k-1), \ldots, \rho(k), \rho(k-1), \ldots] \in S_{u}
$$

that (i) renders the origin an exponentially stable equilibrium point of the closed loop system, and (ii) at each time $k$ it minimizes the following performance index:

$$
J[\rho(k), u]=\sup _{\rho \in \mathcal{F}_{\Theta}} \mathbf{E}\left\{\frac{1}{N} \sum_{t=k}^{k+N-1} z^{T}(t) z(t)\right\}
$$

Remark 1: The horizon $N$ is a parameter than can be use to tune transient versus steady-state behavior. A reasonable value for $N$ is the minimum amount of time needed for the parameter trajectories $\rho($.$) to transition between any 2$ arbitrary points in $\mathcal{P}$, since the parameter value at time $t$ carries no information about its value beyond $t+N$. Hence beyond this horizon the problem reverts to a robust optimization over all possible parameter values.

In the sequel, for simplicity, we make the following standard assumptions: $D_{12}^{T} D_{12}=I, C_{1}^{T} D_{12}=0$, and $\mathbf{E}\left(w_{k} w_{k}^{T}\right)=I$. In addition, the explicit dependence of matrices on $\rho$ will be omitted, when it is clear from the context.

\section{B. Preliminary Results}

In this section we introduce some definitions and preliminary results that will be used to reduce the minimization of (4) to a tractable finite dimensional optimization. For ease of reading, all proofs in this section are relegated to the Appendix.

Definition 1: A set $S_{x} \doteq\left\{x \in R^{n_{x}}: F(x) \leq 1\right\}$ is said to be mean controlled invariant for the system (1) if, given an initial condition $x_{o} \in S_{x}$ there exist a sequence of admissible control actions $\left\{u_{i}\right\}, u_{i} \in S_{u}$ such that the corresponding trajectory satisfies $\mathbf{E}\{F[x(k)]\} \leq 1$, for all $k>0$, where the expectation is taken with respect to the input sequence $w($.$) .$
Lemma 1: Consider an exponentially stable Linear Time Varying (LTV) system of the form:

$$
\begin{aligned}
x(t+1) & =A(t) x(t)+B(t) w(t) \\
z(t) & =C(t) x(t)+D(t) w(t)
\end{aligned}
$$

with a Gaussian distributed initial condition $x_{o}$ with covariance $\mathbf{E}\left(x_{o} x_{o}^{T}\right)=X_{o}$. Then, for any $X(t) \geq 0$ satisfying the affine matrix inequality

$$
A(t) X(t) A^{T}(t)-X(t+1)+B(t) B^{T}(t) \leq 0
$$

with initial condition $X(0) \geq X_{o}$ the following bound holds:

$$
\begin{array}{r}
\mathbf{E}\left\{\frac{1}{T} \sum_{t=0}^{T-1} z^{T}(t) z(t)\right\} \leq \frac{1}{T} \sum_{t=0}^{T-1} \operatorname{Trace}\left[C(t) X(t) C^{T}(t)\right. \\
\left.+D(t) D^{T}(t)\right]
\end{array}
$$

Similarly, if $Y(t) \geq 0$ satisfies:

$$
A^{T}(t) Y(t+1) A(t)-Y(t)+C^{T}(t) C(t) \leq 0
$$

then

$$
\begin{aligned}
& \mathbf{E}\left\{\frac{1}{T} \sum_{t=0}^{T-1} z^{T}(t) z(t)\right\} \leq \text { Trace }\left[X_{o} Y(0)\right]+ \\
& +\frac{1}{T} \sum_{t=0}^{T-1} \operatorname{Trace}\left[B^{T}(t) Y(t+1) B(t)+D^{T}(t) D(t)\right]
\end{aligned}
$$

Next, we show that the $\mathcal{H}_{2}$ cost can be partitioned into a portion related to a state-feedback problem for an auxiliary plant, plus an "observation" cost. These results can be though of as an extension to the LPV case of some general orthogonality results originally introduced in [16] for the LTI case.

Theorem 1: Consider an LPV system of the form (1) and define the following auxiliary state-feedback plant:

$$
G_{s f}\left\{\begin{aligned}
x_{s f}(t+1) & =A[\rho] x_{s f}(t)-L[\rho] R_{c}[\rho] w(t)+B_{2}[\rho] u \\
z_{1}(t) & =C_{1}[\rho] x_{s f}(t)+D_{12}[\rho] u(t) \\
y(t) & =x_{s f}(t)
\end{aligned}\right.
$$

where

$$
\begin{aligned}
L & =-A Z^{-1} C_{2}^{T} R_{c}^{-2} \\
R_{c} & =\left(I+C_{2} Z^{-1} C_{2}^{T}\right)^{\frac{1}{2}}
\end{aligned}
$$

and where $Z()>$.0 satisfies the following affine matrix inequality:

$$
\left[\begin{array}{ccc}
-Z(\theta) & Z(\theta) A(\rho) & Z(\theta) B_{1}(\rho) \\
A^{T}(\rho) Z(\theta) & -Z(\rho)-C_{2}^{T}(\rho) C_{2}(\rho) & 0 \\
B_{1}^{T}(\rho) Z(\theta) & 0 & -I
\end{array}\right]<0
$$

for all $\rho \in \mathcal{P}$ and $\theta \in \Theta(\rho)$. Finally, let $K[\rho]$ be such that $A[\rho]+B_{2}[\rho] K[\rho]$ is exponentially stable for all admissible parameter trajectories and consider the following output feedback controller:

$$
\begin{aligned}
x_{c}(t+1) & =\left(A[\rho]+B_{2}[\rho] K[\rho]+L[\rho] C_{2}[\rho]\right) x_{c}(t)-L[\rho(t)] y(t) \\
u(t) & =K[\rho] x_{c}(t) \quad x_{c}(0)=0
\end{aligned}
$$

and denote by $G_{c l}$ and $z_{c l}$ the system obtained by closing the loop around (1) using the controller (13), and its corresponding output, respectively. Then (i) $G_{c l}$ is exponentially stable and, (ii) The corresponding closed loop trajectories satisfy

$$
\mathbf{E}\left[z^{T}(t) z(t)\right] \leq \mathbf{E}\left(z_{s f}^{T} z_{s f}\right)+\text { Trace }\left[C_{1}(t) Z^{-1}(t) C_{1}^{T}(t)\right]
$$


where $z_{s f}$ denotes the output of $G_{s f}$ corresponding to the control law $u_{s f}=-K[\rho] x_{s f}$.

\section{Risk Adjusted RECEDING HoRIZON CONTROL: THE UNCONSTRAINED CONTROL CASE}

\section{A. Motivation:}

In this section we introduce a conceptual receding horizon control law for unconstrained LPV systems that provides the motivation for the risk-adjusted approach pursued latter in the paper. In principle, one could synthesize a receding horizon control law by exploiting Theorem 1 to reduce the problem to an equivalent state feedback one, which in turn can be reduced, via Lemma 1 to a (functional) convex optimization over the set of matrix functions of $\rho$. However, solving this problem is far from trivial, even when making several approximations such as assuming memoryless, arbitrarily fast time-varying parameters ${ }^{1}$, typically requiring both gridding of the parameter set $\mathcal{P}$ and approximating $Y($.$) by a finite expansion in order to get a tractable, finite$ dimensional problem [1]. To avoid this difficulty, motivated by the work in [22], [21], in the sequel we will search over closed-loop strategies, rather than control actions. To this effect, denote by $\mathbf{X}_{N+1}: \Theta \times\{-1,0,1, \ldots, N-1\} \rightarrow P$ and $\mathbf{V}_{N+1}: \Theta \times\{-1,0,1, \ldots, N-1\} \rightarrow P$ the set of all bounded matrix functions that map $N+1$-length admissible parameter trajectories to $P$, the class of all symmetric positive definite matrices and $V$, the class of all $n_{u} \times n_{x}$ matrices respectively, and consider the following receding horizon type control law:

Algorithm 1:

0.- Set $x_{c}(0)=0, \tilde{X}(0)=\tilde{W}(0)=\mathbf{E}\left(x_{o} x_{o}^{T}\right)$, and $n=1$.

1.- Let $\rho(n)$ denote the measured value of the parameter at time $\mathrm{n}$ and solve the following LMI optimization problem in $X, \mathcal{W}, \Gamma_{i}$ :

$$
\min _{\mathcal{X}, \mathcal{W} \in \mathbf{X}_{N+1}} \text { Trace }\left\{\frac{1}{N} \sum_{i=0}^{N-1} \Gamma_{i}\right\}
$$

subject to:

$$
\begin{array}{r}
M(\rho) \doteq\left[\begin{array}{ccc}
M_{11} & D_{12}(n+i) V(i) & C_{1}(n+i) \\
V^{T}(i) D_{12}^{T}(n+i) & -X(i) & 0 \\
C_{1}^{T}(n+i) & 0 & -W(i)
\end{array}\right] \leq 0 \\
R(\rho) \doteq\left[\begin{array}{cc}
R_{11} & R_{12} \\
R_{12}^{T} & -X(i-1)
\end{array}\right]<0 \\
S(\rho) \doteq\left[\begin{array}{ccc}
-W(i) & S_{12} & S_{13} \\
S_{12}^{T} & S_{22} & 0 \\
S_{13}^{T} & 0 & -I
\end{array}\right]<0,
\end{array}
$$

\footnotetext{
${ }^{1}$ In this case the problem becomes essentially equivalent to synthesizing a controller for a system subject to parametric uncertainty, and it is well known that these problems are generically NP hard.
}

where:

$$
\begin{aligned}
& M_{11}=-\Gamma_{i}+C_{1}(n+i) X(i) C_{1}^{T}(n+i), \\
& R_{11}= A(n+i-1) X(i-1) A^{T}(n+i-1) \\
&+B_{2}(n+i-i) V(i-1) A^{T}(n+i-1) \\
&+A(n+i-1) V^{T}(i-1) B_{2}^{T}(n+i-1) \\
&+B_{1}(n+i-1) B_{1}^{T}(n+i-1)-X(i), \\
& R_{12}= B_{2}(n+i-1) V(i-1), \\
& S_{12}= W(i) A[\rho(n+i-1)], S_{13}=W(i) B_{1}[\rho(n+i-1)], \\
& S_{22}=-W(i-1)-C_{2}^{T}[\rho(n+i-1)] C_{2}[\rho(n+i-1)] ; \\
& \text { for all } \rho(n+i+1) \in \Theta[\rho(n+i)], i=0,1, \ldots, N-1
\end{aligned}
$$

with boundary conditions $X(-1)=\tilde{X}(n-1)$ and $W(-1)=\tilde{W}(n-1)$, where, with a slight notational abuse, we denote $X[\rho(t), t]$ and $W[\rho(t), t]$ simply as $X(t), W(t)$.

2.- $\operatorname{Set} \tilde{X}(n)=X(0), \tilde{W}(n)=W(0), \tilde{V}(n)=V(0)$ and compute:

$$
\begin{aligned}
L(n)= & -A(n) \tilde{W}^{-1}(n) C_{2}^{T}(n) R_{c}^{-2}(n) \\
K(n)= & \tilde{V}(n) \tilde{X}^{-1}(n) \\
x_{c}(n+1)= & {\left[A(n)+L(n) C_{2}(n)+B_{2} K(n)\right] x_{c}(n) } \\
& -L(n) y(n)
\end{aligned}
$$

3.- Use as control action at time $n$

$$
u_{R H}[\rho(n)]=\tilde{V}[\rho(n)] \tilde{X}^{-1}[\rho(n)] x_{c}(n)
$$

Set $n=n+1$ and go to step 1 .

Theorem 2: The control law $u_{R H}$ renders the origin an asymptotically stable equilibrium point of (1). Moreover the corresponding closed loop trajectory satisfies:

$$
\begin{aligned}
J_{\text {worst }}[\rho(n)] & \doteq \sup _{\rho \in \mathcal{F}_{\Theta}} \mathbf{E}\left\{\frac{1}{N} \sum_{t=n}^{n+N-1} z^{T}(t) z(t)\right\} \\
& \leq \text { Trace }\left\{\sum_{t=n}^{n+N-1} \Gamma_{t}\right\}
\end{aligned}
$$

Proof: Let $G_{s f, c l}$ denote the closed loop system obtained by closing the loop around $G_{s f}$ using the full state feedback control law $u=\tilde{V} \tilde{X}^{-1} x$. A Schur complement argument shows that the inequality (17) implies:

$$
A_{K}^{T}(t-1) \tilde{X}^{-1}(t) A_{K}(t-1)-\tilde{X}^{-1}(t-1)<0
$$

where $A_{K} \doteq A+B_{2} \tilde{V} \tilde{X}^{-1}$. Moreover, compactness of $\mathcal{P}$, together with continuity of all matrices involved, implies that there exist some constants $c_{1}, c_{2}$ such that $c_{1} I \geq \tilde{X}^{-1} \geq c_{2} I$. Hence $x^{T} \tilde{X}^{-1}[\rho] x$ is a parameter dependent Lyapunov function for the plant $G_{s f, c l}$, which implies that $x \rightarrow 0$ as $t \rightarrow \infty$. Moreover, this convergence is uniform, since all matrices involved are functions of $t$ only through $\rho$. Exponential stability of $G_{s f, c l}$ follows now from the equivalence between uniform asymptotic and exponential stability for linear systems ([10], Theorem 3.9). Finally, the inequality (18) implies that $A_{L} \doteq A+L C_{2}$ is exponentially stable (see the proof of Lemma 3 in the Appendix). Exponential stability of 
the plant $G$ when using (20) follows now from Theorem 1 and Lemma 3. Finally, note that

$$
\begin{aligned}
& \operatorname{Trace}\left\{\left(C_{1}+D_{12} K\right) X\left(C_{1}+D_{12} K\right)^{T}\right\} \\
& =\operatorname{Trace}\left\{X\left(C_{1}+D_{12} V X^{-1}\right) X\left(C_{1}+D_{12} V X^{-1}\right)^{T}\right\} \\
& =\operatorname{Trace}\left\{C_{1} X C_{1}^{T}+D_{12} V X^{-1} V^{T} D_{12}^{T}\right\}
\end{aligned}
$$

where we made use of the fact $C_{1} D_{12}^{T}=0$. Hence, the inequality (16) implies that, for all parameter trajectories compatible with the present value of the parameter we have: $\sum_{i=0}^{N-1} C_{c l}(n+i) X(i) C_{c l}^{T}(n+i)+C_{1}(n+$ i) $W^{-1}(i) C_{1}^{T}(n+i) \leq \sum_{i=0}^{N-1} \Gamma_{i}$, where $C_{c l} \doteq C_{1}+D_{12} K$. The bound in (21) follows from Theorem 1 and Lemma 1.

\section{B. Reducing the computational complexity}

As shown in Theorem 2, the receding horizon control law (20) is guaranteed to stabilize the system while minimizing a performance index that takes into account both the transient and steady state behavior. However, in principle it has a high computational complexity, since it requires finding feasible solutions to the set of LMIs (16)-(18). To avoid this difficulty, in the sequel we propose to pursue a a stochastic approximation approach, whose complexity grows polynomially (rather than exponentially) with the horizon $N$. To this effect, assume that $\hat{\rho}=[\rho(n+1) \rho(n+2) \cdots \rho(n+N)]$ has a non-zero probability density for all $\rho \in \mathcal{F}_{\theta}$. Next, note that to compute $u(n)$, one only needs $X(0), W(0)$ and $V(0)$. This observation allows for reformulating the optimization problem (15) to eliminate the need to explicitly compute $X(i), W(i)$ and $V(i), i=1, \ldots, N-1$ as follows: Given a fixed instant $n, \rho(n)$ and $\hat{\rho} \in \mathcal{F}_{\theta}$, define

$$
\bar{M}(n) \doteq\left[\begin{array}{ccc}
\tilde{M} & & \\
& \tilde{R} & \\
& & \tilde{S}
\end{array}\right]
$$

where

$$
\tilde{T}=\left[\begin{array}{lll}
T[\rho(n)] & & \\
& \ddots & \\
& & T[\rho(n+N-1)]
\end{array}\right], T=M, R, S .
$$

In terms of $\bar{M}$, the constraints (16)-(18) can be expressed as:

$$
\begin{aligned}
& \underline{\lambda}\left(\Gamma_{0}, \ldots \Gamma_{N-1}, X(0), W(0), V(0), \rho(n), \hat{\rho}\right) \doteq \\
& \quad \min \quad \lambda_{\max }[\bar{M}(n)]<0 . \\
& W(1), \ldots, W(N-1) \\
& V(1), \ldots, V(N-1)
\end{aligned}
$$

Thus, in this context, one does not need to compute the explicit value of $X(i), W(i)$ and $V(i), i=1, \ldots, N-1$, as long as the minimum above can be computed. Note in passing, that this reformulation preserves convexity, since the function $\underline{\lambda}(\cdot)$ is a convex function of its arguments. To complete the approximation of the original optimization problem by a stochastic one, given $\zeta>0$, define

$$
\tilde{g}(x)=\frac{e^{\zeta x}-1}{\zeta}
$$

and collect all the optimization variables (e.g., the entries of $\Gamma_{0}, \ldots, \Gamma_{N-1}, X(0), W(0)$ and $\left.V(0)\right)$ in a vector $\mathbf{x}_{n}$. Define the following functions

$$
\begin{gathered}
f_{0}\left(\mathbf{x}_{n}, \rho(n), \hat{\rho}\right) \doteq \operatorname{Trace}\left\{\frac{1}{N} \sum_{i=0}^{N-1} \Gamma_{i}\right\} \\
f_{1}\left(\mathbf{x}_{n}, \rho(n), \hat{\rho}\right) \doteq \tilde{g}[\underline{\lambda}(\Gamma, X(0), W(0), V(0), \rho(n), \hat{\rho})]+\varepsilon .
\end{gathered}
$$

Consider now the following convex problem

$$
\begin{aligned}
& \min \mathbf{E}_{\hat{\rho}}\left[f_{0}\left(\mathbf{x}_{n}, \rho(n), \hat{\rho}\right)\right]=\min \operatorname{Trace}\left\{\frac{1}{N} \sum_{i=0}^{N-1} \Gamma_{i}\right\} \\
& \text { s.t. } \mathbf{E}_{\hat{\rho}}\left[f_{1}\left(\mathbf{x}_{n}, \rho(n), \hat{\rho}\right)\right] \leq 0 ;
\end{aligned}
$$

where $\mathbf{E}_{\hat{\rho}}[\cdot]$ denotes the expected value with respect to the random variable $\hat{\rho}$. It can be easily shown that the solution to this problem tends to the solution of the problem (15) as $\zeta \rightarrow \infty$ and $\varepsilon \rightarrow 0$. We are now ready to provide the main result of this section: an algorithm for solving problem (24) in polynomial time. For technical reasons, in the sequel we will assume that the solution to this problem is known to belong to a given compact convex set $X$ (where the matrices $\Gamma_{0}, \ldots, \Gamma_{n-1}, X(0), W(0)$ and $V(0)$ have bounded entries and $X(0)$ and $W(0)$ are positive definite).

Let $\pi(\cdot)$ denote the projection onto $X$; i.e.,

$$
\pi(\mathbf{x})=\arg \min _{\tilde{\mathbf{x}} \in X}\|\mathbf{x}-\tilde{\mathbf{x}}\|_{2}
$$

and consider the following algorithm:

Algorithm 2:

1.- Initialization: Determine $\mathbf{x}_{n}^{0}, y_{i}^{0} ; i=0,1$ and $z_{1}^{0}$. Let $k=0$.

2.- Generate a sample $\hat{\rho}^{k}=\left[\rho^{k}(n+1), \ldots, \rho^{k}(n+N-1)\right]$

3.- If $z_{1}^{k} \leq-\gamma_{0} / k^{\gamma}$ then

$$
\mathbf{x}_{n}^{k+1}=\pi\left[\mathbf{x}_{n}^{k}-b_{k} y_{0}^{k}\right]
$$

4.- Let

Otherwise, $\mathbf{x}_{n}^{k+1}=\pi\left[\mathbf{x}_{n}^{k}-b_{k} y_{1}^{k}\right]$.

$$
y_{i}^{k+1}=y_{i}^{k}+a_{k}\left(\left.\frac{\partial f_{i}\left(\mathbf{x}_{n}, \rho(n), \hat{\rho}^{k}\right)}{\partial \mathbf{x}_{n}}\right|_{\mathbf{x}_{n}=\mathbf{x}_{n}^{k}}-y_{i}^{k}\right)
$$

$i=0,1$ and

$$
z_{1}^{k+1}=z_{1}^{k}+a_{k}\left(f_{1}\left(\mathbf{x}_{n}, \rho(n), \hat{\rho}^{k}\right)-z_{1}^{k}\right) ;
$$

5.- If $z_{1}^{l}<0$ and $\left|f_{0}\left(\mathbf{x}_{k}, \rho(n), \hat{\rho}\right)-f_{0}\left(\mathbf{x}_{k-1}, \rho(n), \hat{\rho}\right)\right|<\varepsilon_{o}$ for $l=k-N_{\text {good }}+1, \ldots, k+1$ stop. Otherwise, let $k=$ $k+1$ and go to step 3 .

Theorem 3: Let $a_{k}=\frac{\alpha_{0}}{k^{\alpha}} ; b_{k}=\frac{\beta_{0}}{k^{\beta}}$, where $\alpha_{0}, \alpha, \beta_{0}$ and $\beta$ are positive constants. Furthermore, assume that $\gamma_{0}$ and $\gamma$ are also positive. Then, if

$$
\sum_{k=0}^{\infty} a_{k}=\sum_{k=0}^{\infty} b_{k}=\infty ; \quad \sum_{k=0}^{\infty} a_{k}^{2}<\infty ; \lim _{k \rightarrow \infty} \frac{b_{k}}{a_{k}}=0
$$

and $2 \beta-\alpha-2 \gamma>1$ the sequence $\mathbf{x}_{n}^{k}$ converges with probability one to the solution of the problem (24).

Proof: Direct application of Theorem 1 in [8]. 


\section{Adding Control Constraints}

In this section we briefly indicate how to modify the algorithm presented above to handle constraints in the control action. In the case of deterministic systems this can be accomplished by suitably scaling the control action using state dependent weights [20], [21]. However, this approach cannot be directly applied here, since the stochastic nature of system (1) prevents predicting the future values of these scalings, or, equivalently rendering suitable sets controlledinvariant. To circumvent this difficulty, in this paper we propose to replace the constraints $u(n+i) \in S_{u} \forall i=0,1, \ldots N-1$ in the optimization of (4) by the relaxed set $u(n) \in S_{u}$, $\mathbf{E}[u(n+i)] \in S_{u}, i=1, \ldots, N-1$. That is, at each given time $n$, the present value of the control action is subject to the hard constraint $u(n) \in S_{u}$, while only the expected value of predicted future control actions $u(n+1), \ldots, u(n+N-1)$ is required to satisfy the constraints.

Specifically, assume that the control constraint set is of the form $S_{u}\left\{u:\|u\|_{\infty} \leq 1\right\}$. When using a control law of the form (20), this constraint is equivalent to

$$
\left|u_{j}\right| \leq 1 \Longleftrightarrow\left|v_{j} X^{-\frac{1}{2}} X^{-\frac{1}{2}} x\right| \leq 1, \quad j=1, \ldots, n_{u}
$$

where $v_{j}$ denotes the $j^{\text {th }}$ row of the matrix $V$. This can be relaxed to:

$\left|u_{j}\right| \leq 1 \Leftarrow\left(v_{j} X^{-1} v_{j}^{T}\right) \cdot\left(x^{T} X^{-1} x\right) \leq 1 \Leftarrow\left\{\begin{array}{c}v_{j} X^{-1} v_{j}^{T} \leq \frac{1}{c} \\ x^{T} X^{-1} x \leq c\end{array}\right.$

where $c>0$ is an arbitrary constant. We propose to further relax this constraint for $u(n+i)$ to:

$$
\mathbf{E}\left\{\left|u_{j}(n+i)\right|\right\} \leq 1 \Leftarrow\left\{\begin{array}{r}
v_{j} X^{-1} v_{j}^{T} \leq \frac{1}{c} \\
\mathbf{E}\left(x^{T} X^{-1} x\right) \leq c
\end{array}\right.
$$

Assume next that $X$ is chosen so that the closed-loop system satisfies (6) with $X(0) \geq X_{o}$. Then the corresponding closed loop trajectories satisfy $X(n+i) \geq \mathbf{E}\left\{x(n+i) x^{T}(n+i)\right\}$. Hence

$$
\begin{aligned}
& \mathbf{E}\left[x^{T}(n+i) X^{-1}(n+i) x(n+i)\right] \\
& =\operatorname{Trace}\left\{X^{-1}(n+i) \mathbf{E}\left[x(n+i) x^{T}(n+i)\right]\right\} \\
& \leq \operatorname{Trace}\left\{I_{n_{x}}\right\}=n_{x}
\end{aligned}
$$

It follows that the ellipsoid $x(n+i)^{T} X^{-1}(n+i) x(n+i) \leq n_{x}$ is mean controlled invariant and thus the control constraints (26) can be enforced by simply enforcing the constraint $v_{j} X^{-1} v_{j}^{T} \leq \frac{1}{n_{x}}$. These observations lead to the following Receding Horizon algorithm:

\section{Algorithm 3:}

0.1 - Set $x_{c}(0)=0, \tilde{X}(0)=\tilde{W}(0)=\mathbf{E}\left\{x_{o} x_{o}^{T}\right\}$ and $n=1$.

1.- Let $\rho(n)$ denote the measured value of the parameter at time $\mathrm{n}$ and solve the following LMI optimization problem in $X, \mathcal{W}, \mathcal{V}$ and $\Gamma_{i}$ :

$$
\begin{gathered}
\min _{\substack{\mathcal{W} \in \mathbf{X}_{N+1} \\
\mathcal{V} \in \mathbf{V}_{N+1}}} \operatorname{Trace}\left\{\frac{1}{N} \sum_{i=0}^{N-1} \Gamma_{i}\right\} \\
\text { subject to (16),(17),(18) and } \\
{\left[\begin{array}{cc}
n_{x} & x_{c}^{T}(n) \\
x_{c}(n) & X(n)
\end{array}\right] \geq 0,\left[\begin{array}{cc}
\frac{1}{n_{x}} & v_{j}(n+i) \\
v_{j}^{T}(n+i) & X(n+i)
\end{array}\right] \geq 0} \\
j=1, \ldots, n_{u}, i=0,1, \ldots, N-1
\end{gathered}
$$

for all $\rho(n+i+1) \in \Theta[\rho(n+i)], i=0,1,2, \ldots, N-1$

2.- Set $\tilde{X}(n)=X(0), \tilde{W}(n)=W(0), \tilde{V}=V(0)$ and compute:

$$
\begin{aligned}
L(n)= & -A(n) \tilde{W}^{-1}(n) C_{2}^{T}(n) R_{c}^{-2}(n) \\
K(n)= & V(n) \tilde{X}^{-1}(n) \\
x_{c}(n+1)= & {\left[A(n)+L(n) C_{2}(n)+B_{2} K(n)\right] x_{c}(n) } \\
& -L(n) y(n)
\end{aligned}
$$

3.- Use as control action at time $n$

$$
u_{R H}[\rho(n)]=\tilde{V}[\rho(n)] \tilde{X}^{-1}(n) x_{c}(n)
$$

Set $n=n+1$ and go to step 1 .

Theorem 4: Assume that the origin is an exponentially stable equilibrium point of $A[\rho()$.$] for all \rho \in \mathcal{F}_{\Theta}$ Then, the control law (31) (i) is admissible, in the sense that it satisfies the control constraints $u(k) \in S_{u}, \forall k$, and (ii) it renders the origin a globally exponentially stable point of the closed loop system for all admissible parameter trajectories.

Proof: From Theorem 1 it follows that we only need to consider the state feedback case. The proof of stability in this case is similar to the proof of Theorem 2, provided that the additional constraints (30) are feasible. Feasibility of these constraints follows from the fact that exponential stability of $A($.$) always allows for scaling$ the matrices $V$ and $X$ so that the constraints hold. Finally, the fact that the control law (31) satisfies the constraints $\left|u_{i}().\right| \leq 1$ follows immediately from (30) and (25).

Remark 2: If the open loop system is not exponentially stable the algorithm above will (under mild controllability and observability hypothesis) locally stabilize the system in some neighborhood of the origin, which can be estimated by computing the sets where the constraints (17) and (30) are feasible.

Remark 3: As before, the computational complexity of the optimization problem above grows exponentially with the horizon, even when approximating $X, V$ and $W$ by finite expansions. However, the stochastic approach used in Algorithm 2 can also be used here, with minimal modifications, to obtain polynomial growth approximations.

\section{ILLUSTRATIVE EXAMPLE}

Consider the discrete time LPV system that has the following system matrices:

$$
A(\rho(t))=\left[\begin{array}{cc}
1.01 & 0.1 \\
0 & 1-0.1 \rho(t)
\end{array}\right]
$$




$$
\begin{gathered}
B_{1}(\rho(t))=\left[\begin{array}{ll}
0.1 & 0 \\
0.2 & 0
\end{array}\right], B_{2}(\rho(t))=\left[\begin{array}{c}
0 \\
0.0787
\end{array}\right]^{\prime} \\
C_{1}(\rho(t))=\left[\begin{array}{cc}
1 & 0 \\
-1 & 0
\end{array}\right], D_{12}(\rho(t))=\frac{1}{\sqrt{2}}\left[\begin{array}{ll}
1 & 1
\end{array}\right]^{\prime} \\
C_{2}(\rho(t))=\left[\begin{array}{ll}
1 & 0
\end{array}\right], D_{21}(\rho(t))=\left[\begin{array}{ll}
0 & 1
\end{array}\right]^{\prime}
\end{gathered}
$$

with admissible parameter set

$$
\mathcal{F}_{\Theta}=\{\rho(t): \rho(t+1) \in[-1,1],|\rho(t)-\rho(t+1)| \leq 0.2, \forall t\} .
$$

It can be verified that the matrix function:

$$
Y(\rho(t))=Y_{0}+Y_{1} \rho(t)+Y_{2} \rho^{2}(t)
$$

with

$$
\begin{aligned}
Y_{0} & =\left[\begin{array}{cc}
0.0339 & -0.0197 \\
-0.0197 & 0.0326
\end{array}\right] \\
Y_{1} & =\left[\begin{array}{cc}
0.0031 & -0.0065 \\
-0.0065 & 0.0100
\end{array}\right] \\
Y_{2} & =\left[\begin{array}{cc}
0.0013 & -0.0024 \\
-0.0024 & 0.0032
\end{array}\right]
\end{aligned}
$$

satisfies the functional matrix inequality:

$$
\left[\begin{array}{ccc}
-Y(\rho)+B_{2}(\rho) B_{2}^{T}(\rho) & Y(\rho) A^{T}(\rho)-B_{2}(\rho) B_{2}^{T}(\rho) & Y(\rho) C_{1}^{T}(\rho) \\
A(\rho) Y(\rho)-B_{2}(\rho) B_{2}^{T}(\rho) & -Y(\theta) & 0 \\
C_{1}(\rho) Y(\rho) & 0 & -I
\end{array}\right] \leq 0
$$

for all $\rho \in \mathcal{P}$ and $\theta \in \Theta(\rho)$. Hence the state feedback gain $K(\rho)=-B_{2}^{T}(\rho) Y^{-1}(\rho)$ provides a suboptimal $\mathcal{H}_{2}$ controller for the pair $\left(A, B_{2}\right)$ (see [1], [21] for details). In addition:

$$
Z(\rho(t))=Z_{0}+Z_{1} \rho(t)+Z_{2} \rho^{2}(t)
$$

with

$$
\begin{aligned}
& Z_{0}=\left[\begin{array}{cc}
7.5801 & -3.9968 \\
-3.9968 & 3.3243
\end{array}\right] \\
& Z_{1}=\left[\begin{array}{cc}
-1.8298 & 1.0069 \\
1.0069 & -0.1874
\end{array}\right] \\
& Z_{2}=\left[\begin{array}{cc}
1.1208 & -0.8476 \\
-0.8476 & 0.8066
\end{array}\right]
\end{aligned}
$$

satisfies (12). Thus a parameter dependent controller of the form (13) exponentially stabilizes the system and gives a bound on the performance index. Next, we compare the performance achieved by this controller, which is essentially the discrete time counterpart of the conventional LPV controllers proposed in [1], against the proposed risk adjusted receding horizon controller.

Experiments were run with different parameter trajectories. The following values were used for the risk adjusted RH controller: $N=10, \zeta=30, \alpha=0.6, \alpha_{0}=1, \beta=1$, $\beta_{0}=10^{-2}, \gamma=0.15$ and $\gamma_{0}=10^{-6}$. At each time instant, at most 100 iterations of Algorithm 2 were used to obtain an approximate solution to (15). Table I compares the performance of the receding horizon and conventional controllers. Here, for comparison purposes we used the cost function $\frac{1}{t_{\text {final }}} \sum_{t=0}^{t_{\text {final }}} z^{T}(t) z(t)$. As shown there, the risk adjusted controller yields roughly a $40 \%$ performance improvement on average.

\begin{tabular}{|c|c|c|c|}
\hline & Max & Min & Mean \\
\hline Conventional & 8.1929 & 2.2943 & 5.0713 \\
\hline Risk Adjusted RH & 5.3897 & 1.0587 & 2.9043 \\
\hline
\end{tabular}

TABLE I

COMPARISON OF RESUlTS

\section{CONCLUSIONS}

Practical tools for synthesizing controllers for LPV systems have emerged relatively recently and are still far from complete. Among others, issues not completely solved yet include non-conservative handling of performance specifications and overall computational complexity.

In this paper we take some steps towards removing these limitations by combining Receding Horizon and riskadjusted control ideas. Motivated by some earlier results on stabilization of LTV and LPV systems [22], [19] the main idea of the paper is to recast the problem into an equivalent (functional) convex optimization by searching over a suitable set of closed-loop strategies. This leads to a globally stabilizing control law that is less conservative than techniques currently used to deal with LPV systems. However, in principle this is achieved at the expense of computational complexity, since this law requires the on-line solution of a set of functional LMIs. We propose to address this difficulty by using a risk-adjusted approach, where in exchange for a slight probability of constraint violation, one obtains a substantial reduction in computational complexity. Moreover, this approach scales polynomially, rather than exponentially, with system size [11], [23].

These results were illustrated with a simple example where a risk-adjusted receding horizon controller was used to control a second order LPV plant. As shown there the proposed risk-adjusted receding horizon controller improves performance vis-a-vis a conventional LPV controller, while substantially reducing the computational effort required by a comparable Receding Horizon controller.

Research is currently under way seeking to extend these results to address the issue of model uncertainty, both parametric and dynamic.

\section{APPENDIX}

PROOFS OF THE RESUlTS IN SECTION II-B

\section{A. Proof of Lemma 1}

Proof of (7) follows immediately by noting that $X(k) \doteq \mathbf{E}\left[x(k) x^{T}(k)\right]$ satisfies the equality in (6) and that $\mathbf{E}\left[z^{T}(k) z(k)\right]=\operatorname{Trace}\left[C(k) X(k) C^{T}(k)+D(k) D_{\tilde{X}}^{T}(k)\right]$. Finally, note that for any $\tilde{X}$ satisfying (6), one has $\tilde{X}(k)-X(k) \geq$ $0, \forall k \geq 0$, from where the bound (7) follows. To prove (9), let $\phi(t, i)$ denote the transition matrix of (5). Then its convolution kernel is given by

$$
h(t, i)=\left\{\begin{array}{cc}
C(t) \phi(t, i+1) B(i) & t>i \\
D(t) & t=i \\
0 & t<i
\end{array}\right.
$$


and

$$
\begin{aligned}
\sum_{t=0}^{T-1} \mathbf{E}\left[z^{T}(t) z(t)\right]= & \operatorname{Trace}\left\{\sum_{t=0}^{T-1} \sum_{k=0}^{T-1} h^{T}(t, k) h(t, k)\right. \\
& \left.+\phi^{T}(t, 0) C(t) C^{T}(t) \phi(t, 0) X_{o}+D^{T}(t) D(t)\right\} \\
= & \sum_{k=0}^{T-1} \operatorname{Trace}\left[B^{T}(k) Y(k+1) B(k)+D^{T}(k) D(k)\right] \\
& + \text { Trace }\left[Y(0) X_{o}\right]
\end{aligned}
$$

where we used the facts that $\mathbf{E}\left[\left(w(k) w^{T}(j)\right]=I \delta(k-j)\right.$ and $\mathbf{E}\left[w(k) x^{T}(i)\right]=0$ and defined

$$
Y(k) \doteq \sum_{t=k}^{T-1} \Phi^{T}(t, k) C^{T}(t) C(t) \Phi(t, k)
$$

Straightforward computations show that $Y(k) \geq 0$ satisfies the equality in (8). As before, given any other solution $\tilde{Y}(k)$ to the inequality (8), from the exponential stability of $A(k)$ it follows that $\tilde{Y}(k)-Y(k) \geq 0$ from where the bound (9) follows.

\section{B. Proof of Theorem 1}

In order to prove this Theorem, we need the following preliminary result establishing a general orthogonality property, valid for any stabilizing controller.

Lemma 2: Consider an LPV system of the form (1) and define the following (disturbance feedforward) auxiliary plant $G_{D F}$ :

$G_{D F}\left\{\begin{aligned} x(t+1) & =A[\rho(t)] x(t)-L R_{c}[\rho(t)] w(t)+B_{2}[\rho(t)] u(t) \\ z_{D F}(t) & =C_{1}[\rho(t)] x(t)+D_{12}[\rho(t)] u(t) \\ y(t) & =C_{2}[\rho(t)] x(t)+R_{c}[\rho(t)] w(t)\end{aligned}\right.$

where $L$ and $R_{c}$ are defined in (11). Then for any strictly proper LPV controller $K$ of the form:

$$
K\left\{\begin{aligned}
x_{c}(t+1) & =A_{c}(t) x_{c}(t)+B_{c}(t) y(t) \\
u(t) & =C_{c}(t) x_{c}(t)
\end{aligned}\right.
$$

the following properties holds:

- (i) $K$ internally internally stabilizes $G$ if and only if it internally stabilizes $G_{D F}$

- (ii) The corresponding closed loop trajectories satisfy

$$
\mathbf{E}\left[z^{T}(t) z(t)\right] \leq \mathbf{E}\left(z_{D F}^{T} z_{D F}\right)+\text { Trace }\left[C_{1}(t) Z^{-1}(t) C_{1}^{T}(t)\right]
$$

Proof: Property (i) follows immediately from the fact that $G$ and $G_{D F}$ share the same triple $\left(A, B_{2}, C_{2}\right)$. To prove property (ii) start by considering the following state space realizations of the closed loop systems $G_{c l}$ and $G_{D F, c l}$ :

$$
G_{c l} \doteq\left(\begin{array}{c|c}
A_{c l} & B_{c l} \\
\hline C_{c l} & 0
\end{array}\right), G_{D F, c l} \doteq\left(\begin{array}{c|c}
A_{c l} & B_{D F, c l} \\
\hline C_{c l} & 0
\end{array}\right)
$$

where

$$
\begin{aligned}
A_{c l} & =\left[\begin{array}{cc}
A & B_{2} C_{c} \\
B_{c} C_{2} & A_{c}
\end{array}\right], B_{c l}=\left[\begin{array}{c}
B_{1} \\
B_{c} D_{21}
\end{array}\right], \\
B_{D F, c l} & =\left[\begin{array}{c}
-L R_{c} \\
B_{c} R_{b}
\end{array}\right], C_{c l}=\left[\begin{array}{ll}
C_{1} & D_{12} C_{c}
\end{array}\right]
\end{aligned}
$$

For each fixed parameter trajectory, let $X_{D F}[\rho(t)] \geq 0$ denote the solution to the equation:

$$
\begin{aligned}
A_{c l}[\rho] X_{D F}[\rho(t)] A_{c l}^{T}[\rho]- & X_{D F}[\rho(t+1)]= \\
& -B_{D F, c l}[\rho] B_{D F, c l}^{T}[\rho]
\end{aligned}
$$

define

$$
X[\rho] \doteq X_{D F}[\rho]+\left[\begin{array}{cc}
Z^{-1}[\rho] & 0 \\
0 & 0
\end{array}\right]
$$

where $Z[\rho]>0$ satisfies (12). Straightforward algebraic manipulations using (11) yield:

$$
A_{c l[\rho]} X[\rho(t)] A_{c l}^{T}[\rho]-X[\rho(t+1)]=-\left[\begin{array}{ll}
F_{11} & F_{12} \\
F_{12}^{T} & F_{22}
\end{array}\right]
$$

where

$$
\begin{aligned}
& F_{11}=L[\rho] R_{c} L[\rho]^{T}+Y[\rho(t+1)]-A[\rho] Y[\rho(t)] A^{T}[\rho] \\
& F_{12}=-L R_{c}^{2} B_{c}^{T}-A[\rho] Y[\rho(t)] C_{2}^{T}[\rho] B_{c}^{T}[\rho] \\
& F_{22}=B_{c}[\rho]\left(R_{c}^{2}-C_{2}[\rho] Y[\rho(t)] C_{2}^{T}[\rho]\right) B_{c}^{T}[\rho]
\end{aligned}
$$

and where we have defined $Y \doteq Z^{-1}$ and made use of the explicit expressions (40). A Schur complement argument shows that (12) is equivalent to

$$
A\left(Z(\rho)+C_{2}^{T} C_{2}\right)^{-1} A^{T}-Z^{-1}[\rho(t+1)]+B_{1} B_{1}^{T}<0
$$

and application of the Matrix Inversion Lemma yields:

$$
\begin{aligned}
& A\left(Z(\rho)+C_{2}^{T} C_{2}\right)^{-1} A^{T}-Z^{-1}[\rho(t+1)]+B_{1} B_{1}^{T}= \\
& A Z^{-1}[\rho(t)] A^{T}-Z^{-1}[\rho(t+1)]+B_{1}[\rho] B_{1}^{T}[\rho]-L R_{c}^{2} L^{T}= \\
& A_{L} Z^{-1}[\rho(t)] A_{L}^{T}-Z^{-1}[\rho(t+1)]+B_{1}[\rho] B_{1}^{T}[\rho]+L L^{T}<0
\end{aligned}
$$

where we have defined $A_{L}=A+L C_{2}$. Substitution of (11) and the second inequality in (45) in (43) yields:

$$
A_{c l[\rho]} X[\rho(t)] A_{c l}^{T}[\rho]-X[\rho(t+1)] \leq-B_{c l}[\rho] B_{c l}^{T}
$$

Since $D_{c l}=0$, from Lemma 1 it follows that, for every admissible parameter trajectory we have:

$$
\begin{aligned}
\mathbf{E}\left\{z^{T}(t) z(t)\right\} \leq & \operatorname{Trace}\left\{C_{c l}[\rho] X[\rho(t)] C_{c l}^{T}[\rho]\right\} \\
= & \operatorname{Trace}\left\{C_{c l}[\rho] X_{D F}[\rho(t)] C_{c l}^{T}[\rho]\right\} \\
& + \text { Trace }\left\{C_{1}[\rho] Z^{-1}[\rho(t)] C_{1}^{T}[\rho]\right\} \\
= & \mathbf{E}\left\{z_{D F}^{T}(t) z_{D F}(t)\right\} \\
& + \text { Trace }\left\{C_{1}[\rho] Z^{-1}[\rho(t)] C_{1}^{T}[\rho]\right\}
\end{aligned}
$$

Next, we extend to the LPV case the well known equivalence (see for instance [24], [18]) between Disturbance Feedforward and State Feedback Problems.

Lemma 3: Given an LPV system of the form (36), with initial condition $x(0)=0$, consider the auxiliary statefeedback plant $G_{s f}$ defined in (10) and let $K[\rho]$ be such that $A[\rho]+B_{2}[\rho] K[\rho]$ is exponentially stable for all admissible parameter trajectories. Finally, denote by $G_{D F, c l}$ and $z_{c l}$ the system obtained by closing the loop around (36) using the controller (13), and its corresponding output, respectively. Then $G_{D F, c l}$ is exponentially stable and, for each fixed parameter trajectory it satisfies:

$$
\mathbf{E}\left\{z_{c l}^{T} z_{c l}\right\}=\mathbf{E}\left\{z_{s f}^{T} z_{s f}\right\}
$$


where $z_{s f}$ denotes the output of $G_{s f}$ corresponding to the control law $u_{s f}=-K[\rho] x_{s f}$

Proof: Let $e(k) \doteq x(k)-x_{c}(k)$ and $\xi(k) \doteq\left[\begin{array}{l}x(k) \\ e(k)\end{array}\right]$.

Straightforward computations show that the closed loop system admits the following state space realization:

$$
\begin{aligned}
\xi(k+1) & =\left[\begin{array}{cc}
A+B_{2} K & -B_{2} K \\
0 & A+L C_{2}
\end{array}\right] \xi(k)+\left[\begin{array}{c}
-L R_{c} \\
0
\end{array}\right] w(k) \\
z(k) & =\left[\begin{array}{ll}
C_{1}+D_{12} K & -D_{12} K
\end{array}\right] \xi(k)
\end{aligned}
$$

A Schur complement argument shows that the last inequality in (45) is equivalent to:

$$
\begin{aligned}
0 & >A_{L}^{T}\left(Z^{-1}[\rho(t+1)]-B_{1} B_{1}^{T}-L L^{T}\right)^{-1} A_{L}-Z[\rho(t)] \\
& \geq A_{L}^{T}(Z[\rho(t+1)]) A_{L}-Z[\rho(t)]
\end{aligned}
$$

which implies that $A_{L}$ is exponentially stable. To show exponential stability of the closed loop system, consider any initial condition $\xi(0) \doteq\left[x^{T}(0) e^{T}(0)\right]^{T}$. Since $A+$ $L C$ is exponentially stable, it follows that $e($.$) is bounded$ and $e(t) \rightarrow 0$ as $t \rightarrow \infty$. Since all the matrices involved are continuous functions of $\rho$ and $P$ is compact, from exponential stability of $A+B_{2} K$ it follows that the system:

$$
x(k+1)=\left(A+B_{2} K\right) x(k)-B_{2} K e(k)
$$

is input to state stable ([10], Page 217). Thus, boundedness of $e($.$) implies that that x($.$) is bounded, and$ the fact that $e(t) \rightarrow 0$ implies that $x(t) \rightarrow 0$ ([10], Lemma 5.6). Moreover, the rate of convergence does not depend on the initial time, since all matrices involved depend on time only through $\rho($.$) . Hence \xi=0$ is a uniformly asymptotically stable equilibrium point of the closed loop system. Exponential stability follows from the equivalence of uniform asymptotic and exponential stability for linear time varying systems ([10], Theorem 3.9). To prove (48) note that $e(k)$ is decoupled from the disturbance input $w$. Hence, since $e(0)=0, e(t) \equiv 0$ which implies that $x_{s f}(t)=x(t)$.

\section{Proof of Theorem 1}

Property (i) follows now the facts that the strictly proper controller (13) stabilizes $G_{D F}$ (Lemma 3) and hence it stabilizes $G$ (Lemma 2). Property (ii) follows from properties (ii) in Lemmas 3 and 2.

\section{ACKNOWLEDGMENTS}

This research was supported in part by NSF under grants ECS-0221562, IIS-0312558, ECS-0501166, and CNS-0519897, and AFOSR under grant FA9550-05-1-0437.

\section{REFERENCES}

[1] G. BALAS et. al. (1997). Lecture Notes, Workshop on Theory and Application of Linear Parameter Varying Techniques, 1997 ACC.

[2] B. R. Barmish and C. M. Lagoa (1997). "The Uniform Distribution: A Rigorous Justification for its Use in Robustness Analysis", Mathematics of Control, Signals and Systems,10, 203-222.

[3] I. Batina, A. A. Stoorvogel and S. Weiland (2001). "Stochastic Disturbance Rejection in Model Predictive Control by Randomized Algorithms," Proc. 2001 ACC, Arlington, Va, pp. 732-737.
[4] G. Calafiore, F. Dabbene and R. Tempo (2000). "Randomized Algorithms for Probabilistic Robustness with Real and Complex Structured Uncertainty," IEEE Trans. on Autom. Contr., 45,12, 2218-2235.

[5] X. Chen and K. Zhou (1997). " A Probabilistic Approach to Robust Control", Proc. 36 IEEE CDC, pp. 4894-4895.

[6] C. E. de Souza and A. Trofino, "Gain-scheduled $\mathcal{H}_{2}$ controller synthesis for linear parameter varying systems via parameter- dependent Lyapunov functions", International Journal of Robust and Nonlinear Control, Volume 16, Issue 5, pp. 243-257, 2006.

[7] Y. Fujisaki, F. Dabbene and R. Tempo (2001) "Probabilistic Robust Design of LPV Control Systems," Proc. 2001 IEEE CDC, Orlando, FL, pp. 2019-2024.

[8] A. M. Gupal (1974). "A Certain Stochastic Programming Problem with Constraints of a Probabilistic Nature," Kibernetika (Kiev), n. 6, pp. $94-100$.

[9] A. Jadbabaie, J. Yu and J. Hauser (1999). "Receding horizon control of the Caltech ducted fan: a control Lyapunov function approach," Proc. 1999 IEEE CCA, pp. 51-56,.

[10] H. Khalil, Nonlinear Systems, $2^{\text {nd }}$ Ed., Prentice Hall, Upper Saddle River, N.J., 1996.

[11] P. Khargonekar and A. Tikku (1996). "Randomized Algorithms for Robust Control Analysis and Synthesis Have Polynomial Complexity", Proceedings of the 35th IEEE CDC, pp. 3470-3475.

[12] M. Kothare, V. Balakrishnan and M. Morari (1996). "Robust Constrained Model Predictive Control Using Linear Matrix Inequalities," Automatica, 32, 10, pp. 1361-1379.

[13] D. Q. Mayne, J. B. Rawlings, C.V. Rao and P. O. M. Scokaert (2000). "Constrained Model Predictive Control: Stability and Optimality," Automatica, 36, 6, pp. 789-814.

[14] L. Ray and R. Stengel (1993). "A Monte Carlo Approach to the Analysis of Control System Robustness", Automatica,29,1, 229-236.

[15] B. T. Polyak and R. Tempo (2000). "Probabilistic Robust Design with Linear Quadratic Regulators," Proc. 39 IEEE CDC, pp. 1037-1042.

[16] M. A. Rotea, "The Generalized $\mathcal{H}_{2}$ Control Problem," Automatica, 29, 2, pp. 373-385, 1993.

[17] J. S. Shamma and M. Athans (1992). "Gain Scheduling: Potential Hazards and Possible Remedies," IEEE. Contr. Sys. Mag., 12, 1, pp. 101-17.

[18] R. S. Sánchez Peña and M. Sznaier, Robust Systems Theory and Applications, Wiley \& Sons, Inc., 1998.

[19] M. Sznaier (1999). "Receding Horizon: An easy way to improve performance in LPV systems," Proc. 1999 ACC," pp. 2257-2261.

[20] M. Sznaier and R. Suarez (2000). "Control of Constrained Systems using Receding Horizon Control Lyapunov Functions," Workshop on Systems with Time-Domain Constraints, Eindhoven University of Technology, Eindoven, The Netherlands.

[21] M. Sznaier, C. Lagoa, A. Stoorvogel and X. Li, "Risk Adjusted Receding Horizon Control of LPV Systems," IFAC Nonlinear Control Symposium (NOLCOS 2004), 2004.

[22] G. Tadmor (1992). "Receding horizon revisited: and easy way to robustly stabilize an LTV system," Systems and Control Letters, 18, pp. 285-294.

[23] R. Tempo, E. W. Bai, and F. Dabbene, F. (1996). "Probabilistic Robustness Analysis: Explicit Bounds for the Minimum Number of Samples," Proc. 35th IEEE CDC, pp. 3424-3428.

[24] K. Zhou, J. C. Doyle and K. Glover, Robust and Optimal Control, Prentice Hall, New Jersey, 1996. 